\title{
The Analysis of AGB Star Photospheres
}

\author{
Verne V. Smith ${ }^{1}$ \\ Dept. of Physics, University of Texas at El Paso, El Paso, TX 79968, \\ $U S A$
}

\begin{abstract}
.
The presence of excess ${ }^{12} \mathrm{C},{ }^{13} \mathrm{C},{ }^{14} \mathrm{~N}, \mathrm{Li}$, and the heavy s-process elements in the photospheres of AGB stars betray the operation of $\mathrm{H}$ and ${ }^{4} \mathrm{He}$-burning, hot-bottom burning, and slow neutron-capture nucleosynthesis. Careful abundance analyses of these various elements provides insight into both nucleosynthesis events and mixing processes which occur deep within the AGB star. Recent results for the s-process are discussed.
\end{abstract}

\section{Introduction}

Analyses of the cooler red giants which populate the asymptotic giant branch (AGB) are of interest for a couple of different reasons. From the standpoint of the evolution of stars and populations of stars, the AGB stars (and their close relatives the post-AGB stars reviewed in this volume by Van Winckel) are the link from the red-giant branch to the white dwarfs (the final resting place of all stars less massive than about $8 \mathrm{M}_{\odot}$ ). The AGB stars are also important within the context of chemical evolution, as nucleosynthesis during this phase of stellar evolution is responsible for a substantial fraction of ${ }^{12} \mathrm{C}$ in the Galaxy, as well as most of the heavy, s-process elements, and probably a sizable percentage of the light element ${ }^{7} \mathrm{Li}$. Here we provide a brief review of recent results for the the s-process elements derived from the photospheric spectra of AGB stars and stars which have been polluted by mass transfer from AGB star photospheres .

\section{The operation of the s-process on the AGB}

As quasi-periodic shell ${ }^{4} \mathrm{He}$-burning occurs during thermal pulses on the AGB, mixing episodes increase the atmospheric $\mathrm{C} / \mathrm{O}$ ratio in these red giants, giving rise to the spectral sequence of $\mathrm{M}$ to $\mathrm{MS}$ to $\mathrm{S}$ to $\mathrm{C}$. Carbon stars have been known for well over a century, while the intermediate $\mathrm{C} / \mathrm{O}$ ratio S-stars were first isolated as a specific stellar type by Merrill (1922) and their spectral cousins the MS stars were discussed by Keenan (1954). This increase in C/O is accompanied, in general, by an increasing s-process overabundance, thus the MS, S, and C stars are heavy-element rich AGB stars.

${ }^{1}$ McDonald Observatory, University of Texas, Austin, TX 79912, USA 
It has been known since the work of Schwarzschild \& Harm (1967) and Sanders (1967) that the preferred astrophysical site for the production of many of the s-process nuclei was in a region associated with the $\mathrm{H}$ - and ${ }^{4} \mathrm{He}$-burning shells found in asymptotic giant branch (AGB) stars. Much of the early work which connected the AGB stellar models to the details of the s-process nucleosynthesis was carried out by Iben and collaborators and is summarized nicely in the review by Iben \& Renzini (1983).

Other warmer, heavy-element rich stellar classes are known; Bidelman \& Keenan (1951) identified the barium (Ba) giants as being heavy-element (Ba and $\mathrm{Sr}$ ) rich, and soon after it was realized that the $\mathrm{CH}$ stars discovered and classified by Keenan (1942) were metal-poor halo relatives of Ba stars. In more recent times, Bond (1974) found subgiant and near main-sequence relatives of the giant $\mathrm{CH}$ and $\mathrm{Ba}$ stars and dubbed them $\mathrm{CH}$ subgiants. Since Bond's discovery, many more true main-sequence s-process rich stars of spectral types $\mathrm{F}$ and $\mathrm{G}$ have been identified: Tomkin et al. (1989) or North, Bertet \& Lanz (1994). Observationally defined s-process rich stars thus span the range in luminosity from main-sequence to extremely luminous AGB giants.

Since the s-process was identified with AGB evolution in the 1960's, the lower luminosity s-process rich $\mathrm{Ba}$ and $\mathrm{CH}$ giants and subgiants defied explanation for many years. McClure, Fletcher \& Nemec (1980) broke the logjam of understanding these diverse groups when they discovered that all $\mathrm{Ba}$ stars were binaries with white-dwarf companions. The solution to the s-process nature of these stars thus centers on mass transfer; when the white dwarf now inhabiting these binary systems was a luminous AGB star and had mixed s-process rich material to its surface, it transferred some of this processed matter to its companion, which is now observed as an s-process rich star. Since the pioneering paper of McClure et al. (1980), follow-up studies (McClure 1984; McClure \& Woodsworth 1990) have confirmed the mass-transfer scenario. In addition, it has been shown that the $40 \%$ of S-stars which do not show the lines of Tc I in their spectra are the cooler relatives of the Ba stars and result from mass transfer (Iben \& Renzini 1983; Smith \& Lambert 1988; Brown et al. 1990; Jorissen et al. 1993). Most recently, a number of the active symbiotic binaries have been identified as C-rich (Schmid \& Nussbaumer 1993; Schmid 1994), while the yellow symbiotic stars AG Dra and BD-21 3873 have both been shown to be s-process rich and, thus, technically Ba stars as well as symbiotics (Smith et al. 1996, 1997). There is now a coherent picture of the s-process rich classes of stars: the N-type carbon stars, along with the MS and S stars with Tc, are bonafide AGB stars which have recently dredged fresh s-process material to their surfaces. The other variety of s-process stars (the $\mathrm{Ba}$ and $\mathrm{CH}$ giants, subgiants, and main-sequence stars, the carbon star dwarfs, and now the C- and s-process rich symbiotic stars) result from mass-transfer in certain binary-star systems.

With so many varieties of stars which exhibit clear signatures of enhancements of the s-process in their abundance patterns, it is not surprising that a great deal of effort has been expended by observational spectroscopists in analyzing the details of s-process nucleosynthesis in the heavy-element rich stars. A number of detailed abundance analyses have been conducted on the various heavy-element rich stars over the last 15 years and can be summarized as follows: for the barium giants we have Tomkin \& Lambert (1983, 1986), Smith (1984), 
Kovacs (1985), Smith \& Lambert (1984), Malaney \& Lambert (1988), and Busso et al. (1995), while the main-sequence Ba stars have been studied by Tomkin et al. (1989) and North et al. (1994). The CH giants have been studied by Vanture (1992a, 1992b). The MS and S stars have been studied by Smith \& Lambert (1990), Plez, Smith \& Lambert (1993), Busso et al. (1995), and Lambert et al. (1995), while the most detailed analysis of the s-process in the carbon stars is still Utsumi (1985). Recent abundance studies have uncovered s-process rich post-AGB stars in the globular cluster $\omega$ Cen (Gonzalez \& Wallerstein 1994), as well as s-process enhancements in the yellow symbiotics (Smith et al. 1996, 1997). Taken together, these abundance studies provide the data with which to probe the details of s-process nucleosynthesis in AGB stars.

One question that is basic to an understanding of the s-process in AGB stars is the nature of the neutron source. Since the realization that the slow capture of neutrons is a distinct stellar nuclear process, two candidate neutron producing reactions have been suggested: ${ }^{13} \mathrm{C}(\alpha, \mathrm{n}){ }^{16} \mathrm{O}$ and ${ }^{22} \mathrm{Ne}(\alpha, \mathrm{n}){ }^{25} \mathrm{Mg}$ (Cameron 1955; Greenstein 1954). These two separate sources have very different characters; the s-process in AGB stars is associated with ${ }^{4} \mathrm{He}$-burning temperatures of $\mathrm{T} \geq 10^{8} \mathrm{~K}$, with the ${ }^{13} \mathrm{C}$ source being active near $1 \times 10^{8} \mathrm{~K}$, while the ${ }^{22} \mathrm{Ne}$ source requires higher temperatures nearer to $2-3 \times 10^{8} \mathrm{~K}$. The different temperatures required to drive the s-process via the different neutron sources will occur in different mass AGB stars, or at different points along the AGB. Historically, the ${ }^{22} \mathrm{Ne}$ source was favored as the most likely s-process candidate because the production of neon-22 can occur quite naturally as the result of successive $\alpha$-captures onto ${ }^{14} \mathrm{~N}$ in the He-burning shell of an AGB star $\left({ }^{14} \mathrm{~N}(\alpha, \gamma){ }^{18} \mathrm{~F}\left(\mathrm{e}^{+}, \nu\right)^{18} \mathrm{O}(\alpha, \gamma){ }^{22} \mathrm{Ne}\right)$, with the initial ${ }^{14} \mathrm{~N}$ itself coming from CNO-cycle H-burning. The carbon-13 neutron source required an extra mixing mechanism to move protons into the He-burning shell to produce ${ }^{13} \mathrm{C}$ via ${ }^{12} \mathrm{C}(\mathrm{p}, \gamma){ }^{13} \mathrm{~N}\left(\beta^{+}, \nu\right){ }^{13} \mathrm{C}$, and it was not clear as to the physical nature of this extra mixing. However, the expected behavior of these neutron sources with metallicity is different and can be tested by the observations.

A convenient parameter to characterize the relative abundance distribution of the s-process is the neutron exposure, $\tau$, defined as

$$
\tau=\int_{0}^{t^{\prime}} \mathrm{N}_{n}(\mathrm{t}) \mathrm{V}(\mathrm{t}) \mathrm{dt}
$$

where $\mathrm{N}_{n}$ is the neutron density and $\mathrm{V}(\mathrm{t})$ the relative velocity of neutrons and nuclei, with the integral taken over the interval of the s-process episode. A large neutron exposure leads to a larger amount of heavier nuclei produced (e.g. Ba, $\mathrm{La}$, or $\mathrm{Ce})$ relative to the lighter nuclei, such as $\mathrm{Y}$ or $\mathrm{Zr}$.

As pointed out by Clayton (1988), the nature of the dominant s-process neutron source can be tested by using the heavy- to light-element abundance distribution of the s-process (a measure of $\tau$ ) as a function of metallicity. For example if, during the s-process episode, the neutrons are in local equilibrium between their production and destruction, then the neutron density will be

$$
\mathrm{N}_{n}=\left(\Sigma \mathrm{N}_{i} \mathrm{~N}_{j}\langle\sigma\rangle_{i, j}\right) /\left(\Sigma \mathrm{N}_{k}\langle\sigma\rangle_{k, n}\right)
$$

where the numerator is summed over all neutron-producing reactions between species $\mathrm{i}, \mathrm{j}$, with Maxwellian-averaged cross sections of $\langle\sigma\rangle_{i, j}$, and the denominator has the neutron destruction (absorbing) reactions, summed over all neutron absorbers, $\mathrm{k}$, and again a Maxwellian-averaged neutron-absorption cross section of $\langle\sigma\rangle_{k, n}$. Making the simplifying assumption that the neutron-producing reac- 


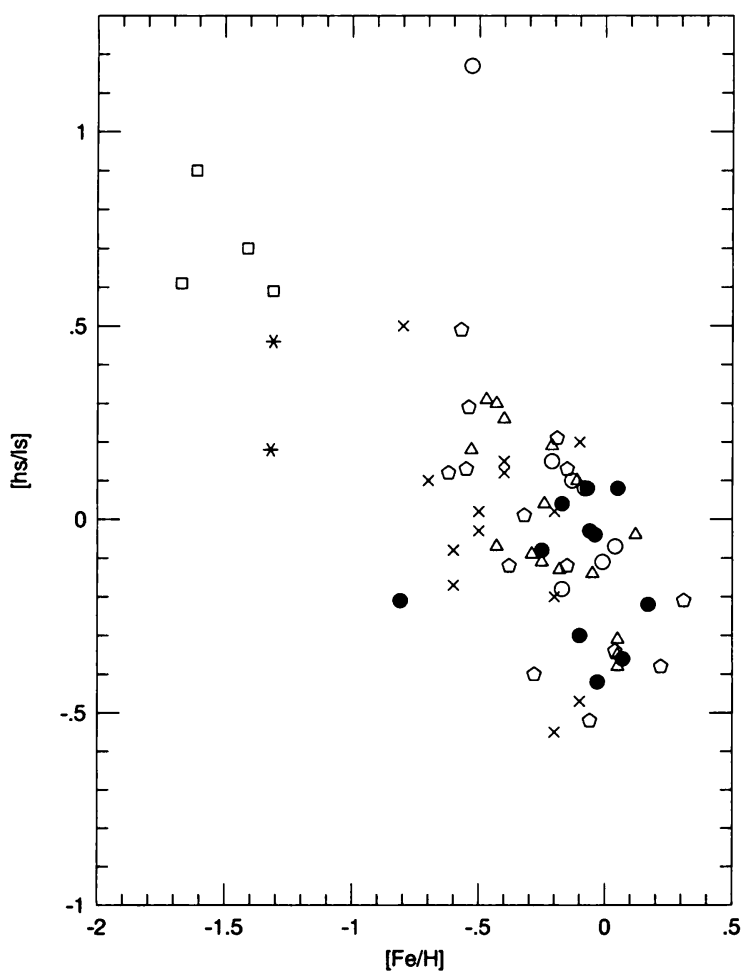

Figure 1. Published abundance results for the heavy s-process elements $(\mathrm{Ba}, \mathrm{La}$ and $\mathrm{Ce}$ ) relative to the light s-process species ( $\mathrm{Y}$ and $\mathrm{Zr}$ ) for the MS/S stars (filled circles, with Tc, and open circles, without $\mathrm{Tc}$ ), $\mathrm{Ba}$ giants (open triangles), $\mathrm{Ba}$ dwarfs (open pentagons), $\mathrm{CH}$ giants (open squares), $\mathrm{CH}$ subgiants (crosses), and yellow symbiotics (six-pointed stars). The increase of heavy to light s-process (and hence the neutron exposure) with decreasing metallicity is clear.

tions come from $\alpha$-captures, and that the dominant neutron absorbers are the seed nuclei of $\mathrm{Fe}$, the above expression can be approximated as

$$
\mathrm{N}_{n} \propto\left(\mathrm{N}_{\mathrm{He}} \mathrm{N}_{j}\right) /\left(\mathrm{N}_{\mathrm{Fe}}\right) \text {, }
$$

where $\mathrm{N}_{j}=\mathrm{N}\left({ }^{13} \mathrm{C}\right)$ for the carbon-13 source and $\mathrm{N}\left({ }^{22} \mathrm{Ne}\right)$ for the neon-22 source. Since the ${ }^{22} \mathrm{Ne}$ in the He-burning layers comes from ${ }^{14} \mathrm{~N}$ which, itself, comes from the initial $\Sigma \mathrm{CNO}$ which is $\propto \mathrm{N}_{F e}$, the expected neutron density (and neutron exposure) would be expected to be roughly independent of metallicity. Carbon13 , on the other hand, must result from the mixing of protons into a ${ }^{12} \mathrm{C}$-rich region, where the carbon- 12 comes from ${ }^{4} \mathrm{He}$, which is almost independent of metallicity. Thus, if the structures of He-shells in AGB stars are not strong functions of metallicity, the numerator of the above expression is constant and $\mathrm{N}_{n}($ and $\tau)$ is $\propto \mathrm{N}_{F e}{ }^{-1}$.

The neutron exposure can be estimated using published abundance results and we show the results of such an exercise in Fig. 1, where we plot the quantity 
[hs/ls] versus $[\mathrm{Fe} / \mathrm{H}]$ from a number of studies. The value of [hs/ls] stands for heavy-s to light-s and is an average overabundance of representative heavy sprocess nuclei ( $\mathrm{Ba}, \mathrm{La}$ and $\mathrm{Ce}$ ) relative to an average overabundance of the light s-process species $\mathrm{Y}$ and $\mathrm{Zr}$, measured in standard spectroscopic bracket notation. There is a rather large scatter, although this is not surprising as we are comparing results from a large number of investigators using somewhat different techniques and different quality data. Nonetheless, there is a clear and significant trend of increasing [hs/ls] with decreasing metallicity.

The increasing value of [hs/ls] indicates a significant increase of neutron exposure with decreasing metallicity as predicted from Clayton's (1988) simple argument for the ${ }^{13} \mathrm{C}(\alpha, \mathrm{n})$ neutron source. Based on the observations, it would appear that the current best-candidate for the s-process neutron source in the AGB stars is carbon-13.

Acknowledgments. This research is sponsored in part by the National Science Foundation (AST96-18459).

\section{References}

Bidelman W.P., Keenan P.C., 1951, ApJ 114, 473

Bond H.E., 1974, ApJ 194, 95

Brown J.A., Smith V.V., Lambert D.L., Dutchover E., Jr., Hinkle K.H., Johnson H.R., 1990, AJ 99, 1930

Busso M., Lambert D.L., Beglio L., Gallino R., Raiteri C.M., Smith V.V., 1995, ApJ 446, 775

Cameron A.G.W., 1955, ApJ 121, 144

Clayton D.D., 1988, MNRAS 234, 1

Gonzalez G., Wallerstein G., 1994, AJ 108, 1325

Greenstein J.L., 1954, in Modern Physics for Engineers, edited by J.L. Ridenour, McGraw-Hill, New York

Iben I., Jr., Renzini A., 1983, ARA\&A21, 271

Jorissen A., Frayer D.T., Johnson H.R., Mayor M., Smith V.V., 1993, A\&A271, 463

Keenan P.C., 1942, ApJ 96, 101

Keenan P.C., 1954, ApJ 120, 484

Kovacs N., 1985, A\&A 150, 232

Lambert D.L., Smith V.V., Busso M., Gallino R., Straniero O., 1995, ApJ 450, 302

Malaney R., Lambert D.L., 1988, MNRAS 235, 695

McClure R.D., 1984, ApJ 280, L31

McClure R.D., Fletcher J.M., Nemec J.M., 1980, ApJ 238, L35

McClure R.D., Woodsworth A.W., 1990, ApJ 352, 709

Merrill P.W., 1922, ApJ 56, 457

North P., Bertet S., Lanz T., 1994, A\&A 281, 775

Plez B., Smith V.V., Lambert D.L., 1993, ApJ 418, 812 
Sanders R.H., 1967, ApJ 150, 971

Schwarzschild M., Harm R., 1967, ApJ 150, 961

Schmid H.M., 1994, A\&A 284, 156

Schmid H.M., Nussbaumer H., 1993, A\&A 268, 159

Smith V.V., 1984, A\&A 132, 326

Smith V.V., Lambert D.L., 1984, PASP 96, 226

Smith V.V., Lambert D.L., 1988, ApJ 333, 219

Smith V.V., Lambert D.L., 1990, ApJS 72, 387

Smith V.V., Cunha K., Jorissen A., Boffin H., 1996, A\&A 315, 179

Smith V.V., Cunha K., Jorissen A., Boffin H., 1997, A\&A 32497

Tomkin J., Lambert D.L., 1983, ApJ 273, 722

Tomkin J., Lambert D.L., 1986, ApJ 311, 819

Tomkin J., Lambert D.L., Edvardsson B., Gustafsson B., Nissen P.E., 1989, A\&A 219, 15

Utsumi K., 1985, in Cool Stars with Excesses of Heavy Elements, edited by M. Jashek and P.C. Keenan, Reidel, Dordrecht, p. 243

Vanture A.D., 1992a, AJ 103, 2035

Vanture A.D., 1992b, AJ 104, 1997 